\title{
Abstracts
}

\section{An Editorial Note}

Social Gerontology is a relatively new enterprise - particularly in Britain - and it draws on a number of academic and professional disciplines. We hope that Ageing and Society will help to forge it in a truly interdisciplinary fashion and the abstracts section represents an important step in this direction. Our aim is to provide readers with summary and comment on articles and rescarch reports relevant to social gerontology but published in journals devoted to other disciplines. Since it would be impossible to scrutinize all relevant journals the review editor identified three subject areas likely to be of particular relevance - Sociology and Social Policy, Social Services, and Psychology and Psychiatry - and invited John Bond (Newcastle), John Tibbitt (Edinburgh) and Mary Gilhooly (Aberdeen) to make their own selections.

This is an experiment rather than a permanent feature and the review editor would welcome comment, suggestions of additional areas and offers from those willing to provide abstracts.

REX TAYLOR

\section{Social Services}

John E. Tibbitt

Challis, D. and Davies, B., 'A New Approach to Community Care for the Elderly', British fournal of Social Work, I0.I, pp. I-I8.

The search for more effective ways of meeting the needs of the elderly has become all the more urgent in the face of rapidly increasing numbers of the 'very elderly' and with the increasing recognition that resources in the forms available hitherto cannot be expanded fast enough nor are particularly appropriate to meet current needs. This paper is concerned with one attempt, the Kent Community Care Project, to provide an alternative approach to meeting the social scrvice needs of those elderly people at or above the margin for residential care.

The paper has two main aims. First, it provides an analysis of the policy context in which the project was conceived, and then goes on to describe the principles of the project to demonstrate how it was envisaged it would 
contribute to the solution of the policy problems. In essence, the Kent project is an attempt to mobilise or generate extra help in the community to meet adequately the needs of some of the vulnerable elderly requiring residential accommodation. Through a decentralized budget given to experienced social workers, fixed at two-thirds of the marginal cost of residential care, workers were able to 'buy in' existing services or negotiate extra help from members of the community for payment, and hence reduce the need for residential care. The paper describes some of the practical problems which arise in implementing such a scheme.

The second aim of the paper is to present some preliminary findings from the evaluation study of the project. The evaluation is based on a quasi-experimental design in which changes in the well-being of the experimental group and a control group drawn from a nearby town with similar socio-demographic characteristics, are compared. The analysis of data for 70 people shows that the experimental group was less likely to become hospitalized at the end of the twelve-month evaluation period and have faired significantly better on a number of variables associated with well-being, at no extra cost to the social services department.

\section{COMMENT}

The Kent approach to community care is an important innovation in service delivery to the elderly which has implications not only in the more efficient use of resources, but also with respect to the role of social workers in the care of the elderly, the flexible integration of different types of service, and the responsiveness of organizational structures to cope with this flexibility and decentralization. Its principles deserve serious consideration by management and practitioners in other localities.

It is encouraging too to see such a scheme being rigorously evaluated: this paper whilst clearly locating the project in its policy objectives is nevertheless rather tantalising in being able to present preliminary evidence from the evaluation. It is to be hoped that the next instalment will not be long delayed.

Kulys, R. and Tobin, S. S., 'Older people and their "responsible other" ', Social Work, 25.2, March 198o, pp. I38-45.

The social problems of many elderly people are often held to be due to their becoming isolated and forgotten by family and friends. This paper, based on research in the US, explores personal relationships between old 
people and those they designate as responsible for their affairs in the event of a crisis, and discusses various aspects of the support system on which elderly people rely. Some implications for the role of social service agencies are then drawn out.

The findings indicate that all but a few respondents could name a 'responsible other'. The individual selected is usually determined by the nature of the kin relationships: spouses were selected before children, children before siblings, and siblings before other family members. If circumstances were such that some deviation from the expected norm was made, respondents were aware of this, implying that the nature of obligation and reciprocity within the kinship system was clear to the elderly.

However, for many in the sample, the relationship with their 'responsible other' was tenuous and fragile, and for others, the 'responsible other' was not the person respondents felt closest to. The authors draw a distinction between the instrumental role in the provision of specific assistance and the affective roles of care and support. They demonstrate that these roles are often scparated within kin networks, or between kin and friendship networks. Of particular concern to the authors are those elderly people, at least ro per cent of the sample, who had a 'responsible other' in name only who fulfilled neither instrumental nor affective functions, and those, some 7 per cent of the sample, who relied on friends who were often elderly themselves, to fulfill both roles.

\section{COMMENT}

Other research has shown that social work with the elderly is frequently seen by practitioners as requiring no particular expertise, cases often being allocated to unqualified workers or to social work assistants. This paper argues that service providers need to be concerned even in a precrisis period with the elderly who lack a supportive 'responsible other', and to concentrate on building or enhancing the support systems of these individuals. Just what a sensitive business this can be is highlighted by the discussion presented here.

Abrams, P., 'Social Change, Social Networks, and Neighbourhood Care', Social Work Service, No. 22, February 1980, pp. 12-23.

In attempting to devclop policies of community care, an important direction in provision for the elderly is a concern with 'good neighbours'. This paper looks at aspects of the reality of neighbourhood life in contemporary 
Britain in order to make some assessment of the prospects for initiatives being undertaken in this field.

At the start attention is drawn to a basic distinction between neighbourhood care as the efficient delivery of formal services where recipients reside and as the cultivation of informal caring activities within neighbourhoods by local residents themselves. It is frequently assumed that these distinct objectives can be reconciled in practice. Most neighbourhood schemes find themselves trying to use formal means to develop informal social relationships and reciprocal care, and find many practical problems as a result.

The core of the paper is concerned with an analysis of 'modern neighbourhoodism' which is distinguished from the traditional neighbourhood as depicted in Bethnal Green and elsewhere, as the context in which neighbourhood care has to be developed. It is argued that the policy problem is not how to prop up or regenerate the traditional neighbourhood but rather to use the features of modern neighbourhoodism, characterized by mobility, choice, organization, and politics, as the social milieu within which informal care can be created.

Neighbourhood care, in both senses of the term, means the working out of a constructive relationship between formal service providers and the neighbourhood movement. The paper suggests three types of relationship which do, or may, arise, namely colonization, conflict, and coexistence. Colonization seems to be the most frequent outcome of present attempts to bridge the boundary between formal and informal services, and can be an effective way of improving neighbourhood care as service delivery. Conflict may easily become a sterile, demoralizing, and hence unproductive process. Under co-existence, the values, norms, and relationships of the informal sector need to be sustained as a basis for policy in their own right and not to suit the purposes of the formal system. This would involve the formal agencies in surrendering some significant powers if a worthwhile measure of informal social care is to be provided within neighbourhood social networks.

\section{GOMMENT}

Social policy is replete with concepts which are broad and ambiguous and whose meaning is rarely analysed in any depth. Nor do the implications of these concepts for the social relationships between those affected by policy receive much attention. This paper is a welcome contribution on both these counts. In bringing a sociological perspective to bear on the notions of neighbourhood and friendship it is able to locate the source of many of the practical problems experienced by current good neighbour schemes, 
and to demonstrate conditions under which neighbourhood care as an informal caring network can develop.

Social Work Services Group, Edinburgh

\section{Sociology and Social Policy}

John Bond

Walker, A., 'The social creation of poverty and dependency in old age', Journal of Social Policy, 9, i98o, pp. 49-75.

This series of abstracts begins with, perhaps, the most relevant article to appear in 1980 . With increasing unemployment, continuing inflation and a deepening recession the living standards of many old people remain below the state's poverty line - the supplementary benefit rate appropriate to each family. From his review of recent data Walker reports that 'one in four elderly people have incomes which are equal to or below the poverty line' (p. 5o).

The central purpose of this article is to explore 'the relationship between poverty in old age and social changes and social policies which have contributed to the social creation of dependency on the state' (p. 51). A large part of the article is taken up with an empirical examination of this relationship. A comprehensive review of secondary data on earnings, incomes and assets, housing circumstances and benefits in kind are presented to show the extent of poverty among old people and to help explain the causes of poverty in old age.

What are the causes of poverty in old age? Walker argues that it has been recognized for a long time that the elderly feature in the lower levels of the income distribution because they are usually not in work. Society rewards present work; it does not reward past work and therefore it does not reward old age. The elderly are discriminated against by economic and social policies which benefit the young and the well-off. He shows that poverty in old age is related to low resources and restricted access to resources throughout the life cycle.

This relationship is well illustrated by describing the process of retirement. Prior to retirement manual workers - the majority of elderly men experience reduced socio-economic status. Increasingly early retirement schemes are pushing elderly workers into less skilled jobs and into unemployment. After retirement the inequalities resulting from low pay, unemployment, disability and for women, sex discrimination, are carried through into old age. The decline in the real value of savings and pensions means that the worst off are the very old. 